Relations industrielles

Industrial Relations

\title{
Current Problems in Labour Arbitration-1978, publié par The Continuing Legal Education Society of B.C., Vancouver, The University of British Columbia, juin 1979, 242 pp.
}

\section{André Roy}

Volume 35, numéro 1, 1980

URI : https://id.erudit.org/iderudit/029050ar

DOI : https://doi.org/10.7202/029050ar

Aller au sommaire du numéro

Éditeur(s)

Département des relations industrielles de l'Université Laval

ISSN

0034-379X (imprimé)

1703-8138 (numérique)

Découvrir la revue

Citer ce compte rendu

Roy, A. (1980). Compte rendu de [Current Problems in Labour Arbitration-1978, publié par The Continuing Legal Education Society of B.C., Vancouver, The University of British Columbia, juin 1979, 242 pp.] Relations industrielles / Industrial Relations, 35(1), 169-171. https://doi.org/10.7202/029050ar

Tous droits réservés @ Département des relations industrielles de l'Université Laval, 1980
Ce document est protégé par la loi sur le droit d'auteur. L'utilisation des services d’Érudit (y compris la reproduction) est assujettie à sa politique d'utilisation que vous pouvez consulter en ligne.

https://apropos.erudit.org/fr/usagers/politique-dutilisation/ 
tionales.

L'aspect novateur, inédit, de cette approche en fait un outil pédagogique de perfectionnement des cadres au niveau international, utile, nécessaire et souhaité.

Dimitri WEISS

Institut d'administration des entreprises, Paris.

\section{Current Problems in Labour Arbitration -} 1978, publié par The Continuing Legal Education Society of B.C., Vancouver, The University of British Columbia, juin 1979, $243 \mathrm{pp}$.

Cette publication miméographiée contient le texte de onze travaux présentés à un séminar tenu en mai et en juin 1978, puis repris en septembre de la même année, sur certaines questions qui se posent au cours d'arbitrage de griefs.

Il n'est pas facile de résumer ces études qui traitent de sujets assez divers et dans lesquelles les auteurs se réfèrent à une jurisprudence volumineuse, non seulement des arbitres, mais aussi des cours civiles.

L'étude liminaire, présentée par D.R. Munroe, président de la Commission des relations de travail de la Colombie Britannique, traite d'une façon générale de l'arbitrage des griefs dans cette province. Le conférencier y souligne les problèmes juridiques et pratiques relatifs à l'arbitrage et il expose le système établi en Colombie Britannique en 1973 et précisé en 1975 lorsqu'a été confié à la Commission le pouvoir de reviser les décisions arbitrales en vue de les soustraire au contrôle des cours civiles. Le pouvoir de révision porte essentiellement sur deux points: le choix des parties à avoir une audition équitable et la conformité des décisions avec les principes énoncés dans le Code du travail. La Commission s'est montrée réservée dans l'exercice de sa compétence de manière que les arbitres ne se sentent pas gênés dans l'exercice de leur liberté et que le recours aux appels ne devienne pas une méthode pour détruire le système. La Commission laisse les arbitres maîtres des faits, puisque ce sont eux qui ont entendu la preuve et elle étudie leurs décisions avec sympathie. Le conférencier estime aussi qu'il faut considérer les arbitrages dans la perspective de l'intérêt public, c'est-à-dire en vue de la diminution des conflits.

La deuxième étude traite d'une question qui se présente dans maints arbitrages: la substitution par l'employeur d'un nouveau motif aux raisons qu'il a originairement fait valoir aux stades antérieurs de la procédure. L'arbitre doit-il permettre cette preuve? Tout dépend des circonstances. En règle générale, les arbitres refusent cette substitution, surtout si le nouveau motif n'a pas été soulevé au comité de griefs. Lorsqu'il acquiesce à la requête de l'employeur, l'arbitre doit accorder un délai à la partie surprise pour étayer sa défense. Cependant, quand se pose la question d'un incident qui serait survenu après celui qui a donné naissance au grief, les arbitres sont enclins à permettre la preuve, sous réserve d'accorder à l'autre partie la possibilité de préparer sa défense.

Le troisième exposé porte sur le droit pour l'employeur d'imposer en cours de convention collective de nouvelles règles disciplinaires ou de nouvelles normes de rendement. En matière de règles disciplinaires, on retient notamment que, pour être acceptées, il faut qu'elles soient compatibles avec la convention collective, qu'elles soient raisonnables et claires, que le salarié en ait été informé, qu'il soit avisé qu'elles peuvent donner lieu à sanctions et qu'elles soient demeurées en vigueur depuis leur introduction. En ce qui concerne les nouvelles normes de rendement, l'on considère que ce droit revient à l'employeur sauf si sa latitude se trouve limitée par la convention collective et pourvu aussi que ces normes nouvelles soient raisonnables et équitables.

Dans un autre exposé, il est traité de la cause juste de congédiement en regard du dossier de l'employé. Ce dossier suit l'employé dans le cheminement de sa carrière et ceci, au moment de l'arbitrage, pose le problème du "culminating incident". Le con- 
férencier étudie la question dans le contexte d'une conduite coupable, d'une conduite sans culpabilité de la part du salarié ou, enfin, dans une situation où l'on retrouve un mélange des deux types de conduite pour conclure que l'on tient généralement compte de deux points: la gravité de la sanction imposée et les délais qui séparent le dernier incident des précédents.

Vient ensuite une étude sur le problème des arbitrages à la suite de sanctions consécutives à une grève illégale ou sauvage. Première question: s'agit-il bien d'une grève ou n'est-ce pas un arrêt de travail attribuable à d'autres causes? La jurise. Première question: s'agit-il bien d'une grève ou n'est-ce pas un arrêt de travail attribuable à d'autres causes? La jurisprudence enseignait autrefois que, peu importe le degré ou la nature de la participation des salariés à une grève illégale, celle-ci était un motif suffisant de congédiement, mais depuis la sévérité des sanctions a été quelque peu tempérée. Ainsi, on en est venu à considérer que le fait d'être un dirigeant syndical n'était pas un motif valable pour le punir plus sévèrement que les autres à moins qu'il n'en ait été un incitateur. L'auteur étudie ensuite trois aspects à retenir dans les griefs de cette nature: l'individualisation des mesures disciplinaires, la sévérité de la sanction imposée et aussi la valeur pratique de ces sanctions.

Un autre conférencier a traité de l'arbitrage des changements technologiques. Il a considéré le problème sous trois aspects. Il s'est demandé d'abord ce que signifie en droit du travail l'expression "changements technologiques» et quelle espèce de modifications elle recourt. Il a fait ensuite une revue de la façon dont les tribunaux d'arbitrage se sont comportés par le passé en regard du droit des entreprises de procéder à de tels changements, en particulier là où la convention collective est silencieuse à ce sujet et il a comparé les jurisprudences canadienne et américaine. Finalement, le conférencier a étudié l'effet de l'insertion dans le Code du travail de la Colombie Britannique de dispositions concernant cette matière des relations professionnelles.

Un conférencier a également traité des arbitrages relatifs aux assignations de travail à des employés qui ne sont pas compris dans l'unité de négociation. La question est ici envisagée sous deux aspects différents: l'assignation de travail à des personnes déjà au service de l'employeur mais qui n'appartiennent pas à l'unité de négociation et, en second lieu, les contrats de sous-traitance. Dans le premier cas, les arbitres ont tendance à tenir compte des dispositions de la convention collective en matière d'ancienneté, de classement des emplois et de taux de salaire et à considérer ces dernières comme autant de restrictions au droit de l'employeur d'agir à sa volonté. En matière de sous-traitance, il existe deux écoles de pensée: l'une qui soutient que, en l'absence de restrictions dans la convention collective, la sous-traitance est un droit strict de l'employeur; l'autre estime que, du seul fait de l'existence de la convention collective, la sous-traitance est prohibée et que, pour que celle-ci soit possible, il appartient à l'employeur de réserver ses droits. L'auteur cite dans son exposé un certain nombre de décisions récentes qui apportent un éclairage nouveau sur ces deux points.

L'étude de la portée des clauses de rétroactivité dans les conventions collectives est aussi un sujet qui a été abordé à ce séminar. Les arbitres doivent se référer aux textes des articles touchant cette question. L'auteur analyse un certain nombre de cas qui se rapportent à des personnes qui ont quitté leur emploi avant l'entrée en vigueur de la convention collective ou encore à l'effet d'une clause générale de rétroactivité qui, se rapportant uniquement aux salaires, ne présentent pas de difficultés insurmontables, il n'en va pas ainsi des dispositions en matière de rétroactivité d'une portée générale qui peuvent parfois confiner à l'absurde ou ne pas rendre l'intention véritable des parties. Les négociateurs, qu'ils soient syndicaux ou patronaux, doivent accorder une grande attention à la rédaction de ces clauses de façon à éviter des différents ultérieurs d'une solution particulièrement difficile. 
D'autres études mériteraient aussi qu'on s'y arrête, en particulier la question de l'extension des unités de négociation et les conséquences, en matière d'arbitrage de l'arrêt de la Cour suprême du Canada dans l'affaire McGavin où le plus haut tribunal du pays a obligé une entreprise à payer à ses employés qu'elle avait congédiés à la suite d'une grève illégale les sommes qui leur étaient dues en vertu d'une clause d'indemnité de départ.

Pour résumer, retenons qu'il s'agit dans cet ouvrage d'un ensemble de textes qui, non seulement sont intéressants, mais peuvent être de grande utilité à ceux qui sont mêlés de près ou de loin aux arbitrages de griefs. En outre d'être une source notable de références, ils permettent de comprendre le fonctionnement du système d'arbitrage en Colombie Britannique, de suivre l'évolution de la pensée des arbitres dans cette province sur des questions quotidiennement soulevées à l'occasion des arbitrages de griefs. La publication du texte de ces travaux ne peut être que bénéfique et stimulante.

\section{André ROY}

Québec

Les aspects humains de l'organisation, par J.L. Bergeron, N. Côté-Léger, J. Jacques et L. Bélanger, Chicoutimi, Gaétan Morin, éditeur, 1979, 337 pp.

Il existe si peu de volumes publiés dans notre milieu et qui traitent de l'application des sciences du comportement aux organisations du travail qu'à ce seul titre, nous n'aurions pu passer sous silence l'ouvrage écrit en collaboration, et qui est paru au dernier trimestre de 1979, par ces quatre universitaires. À leur valeur de pionnier dans le domaine, ils ont également le mérite d'avoir tenté une présentation des principales contributions sectorielles de la psycho-sociologie des organisations ou, ce que plusieurs auteurs nomment, les facteurs humains de l'organisation.
Ainsi, y retrouve-t-on, dans un premier chapitre, qui sert également de cadre intégratif de l'ouvrage, la présentation d'un modèle des déterminants du comportement au sein des organisations. Puis, tour à tour, sont présentés des exposés synthèses sur la personnalité, la perception, les valeurs et attitudes, la motivation, la communication, le groupe (sa nature et son fonctionnement), la coopération et le conflit, le pouvoir, le leadership, le climat organisationnel et la satisfaction au travail et, enfin, l'ouvrage se termine par un chapitre qui ouvre sur le développement organisationnel (D.O.) et la qualité de vie au travail. Avec un menu aussi varié, il est facile de constater que les auteurs se situent dans la lignée la plus classique des ouvrages nordaméricains de la psychologie organisationnelle. Il est d'ailleurs à se demander pourquoi le titre de l'ouvrage n'est pas carrément «Psychologie de l'organisation» ou "Aspects psychologiques du comportement dans les organisations» puisque les aspects sociologiques, économiques, politiques et autres, qui contribuent également aux aspects humains de l'organisation, ont été passés sous silence? C'est peut-être que la trame du volume reflète davantage les intérêts et champs d'intervention des auteurs plutôt que la nature actuelle de ce que sont les aspects humains de l'organisation?

Cet ouvrage présente la forme et la texture d'un recueil ou d'un manuel qui sert à la fois de guide pédagogique et de sources de référence pour le déroulement et le suivi d'un cours. Il nous apparaît comme une suite d'exposés sur les données de recherche et les théories qui ont actuellement cours en psychologie organisationnelle. Le lecteur qui y chercherait une nouvelle approche ou une vue intégrée, originale, adaptée au monde des organisations sortirait probablement déçu de cette lecture. Le fait qu'il ait été conçu et élaboré par plusieurs auteurs accentue l'impression de juxtaposition tant au niveau des données de recherche, de la présentation des théories que du style.

Comme il est mentionné dans le texte de présentation, à l'endos de la couverture, «Les 